\title{
Interactive comment on "Application of Oxygen A-band Equivalent Width for Cloud Optical Depth Measurement” by E. R. Niple et al.
}

\section{Anonymous Referee \#1}

\section{Received and published: 31 March 2016}

The present paper reports on novel application of zenith sky radiance measurements in the visible and near IR spectral ranges with the aim to infer the cloud optical depth (COD). In the core, the method appears sound although it apparently does not deliver unambiguous (and thus very accurate) results. A validation of the method with the AERONET based cloud optical depth retrieval is presented. As such the manuscript could be considered to become published in AMT. The present form the manuscript however comes short in couple of some major points which are outlined below. Therefore I only recommend it for publication after a major revision.

Major comments:

1. Structure of the manuscript: The structure of the manuscript largely deviates from the common form in scientific publishing, i.e. by having a.) a concise abstract, b.) an 
introduction, c.) a section describing the instrument, used methods and tools, followed by $\mathrm{d}$.) a measurement section, and e.) a results and discussion section, and f.) a AMTD concluding section. In particular

- The introduction (as it is) does not describe the state of art, nor does it motivates why a novel instrument and method for cloud optical depth retrievals is necessary or wanted, and what the potential applications are (beyond some very general and not referenced introductory remarks)

- The section describing the instrument, used methods and tools is dispersed over the manuscript so that for example the instrument description appears in a section 7 (rather than upfront in section 2. which could be called 'Instrument description, or Methods\&Tools et cetera

- Finally and in conclusion of 1., it becomes evident that the manuscript is a mixture of a technical description, a collection of some recipes, knowledge and wisdom not referred to existing scientific work, and a minor scientific part. Upgrading the later, however, would be decisive as to whether the paper is suitable to be published in scientific journal.

\section{Physics et cetera}

- Page 2, Line 12: The oxygen A-band is not free from absorption by other species. This is only true but for the spectral region at the lower wavelength end, but at the longer wavelength end water absorption plays role.

- Page 3, line 15: For zenith scattered light, the EQW of the oxygen A-band is known not to uniformly increase with COD. In fact it may even decrease with COD. Only lowlying and optically thick clouds increase the EQW (in the diffuse regime), while upper atmospheric and thinner clouds (cirrus) tend to decrease the EQW (since the optical path is shortened as compared to clear skies).

Interactive

comment 
- Page 3, lines 29 to 32: Your 'Blueness factor' is often (mostly) called color index (google for respective references, there are 100s publications). Beyond this nonstandard use of notion, no further motivation (for example by RT modelling, or by referring to previous work) is given how you arrive at the statement 'a threshold of 5 ' is sure indicator of optically thin clouds'. In a technical description of an instrument, this statement is probably ok without further explanation, but it is certainly insufficient for a scientific paper.

- Why should the inferred COD (measured in the zenith sky for a certain FOV) tightly compare with the cloud mode AERONET data? For example how do the probed cloud areas compare? What are your expectations if they do not totally overlap? Finally why do the AERONET and TWST CODs correlate with a slope of 0.843 ? Again when digging into these questions, scientific work would actually start.

- Page 6, lines 20 to 27: Here the content is totally unclear. What is a bright point radiance, and what is interpolated (I guess the COD as function the maximum radiance in the nose curve?)

- Conclusions: Page 10, lines $27-31$ : Justify why the signal to noise (due to the photo electron shot noise) limits the COD detection? What are the limits of COD detection due to errors and uncertainties in the retrieval. Here you could consult the book of Rodgers 2000 (Inverse methods for atmospheric sounding, Singapore, New Jersey, London, Hongkong: World Scientific.)

3. Terms, notation, and description: Sometimes it becomes difficult to understand what is meant in the manuscript, since often the notation infers from 'laboratory slang'. Just a few examples (out of many). - Title: My strong feeling is that the title of manuscript does not reflect the contents of the paper, since you only use the EQW of the oxygen A-band to provide 'useful information about whether a measurement is in the opticallythin or optically-thick regimes' (page 3 lines 15). In fact you method mostly relies on inspecting the measured zenith radiance at $440 \mathrm{~nm}$ (which is an ambiguous proxy for 
the COD).

- Page 2, line 5: 'absolute power': In radiation physics it would properly be called spectral irradiance, or band integrated irradiance...

- Page 2, line 11: are due to...constituents of the sun: These solar lines are called Fraunhofer lines.

Interactive

comment

- Page 2, line 24: 'Cloud state'. Even though grammatically correct, the proper notation would be 'cloud cover' or 'type of cloud cover'. The notion 'cloud state' could also and easily (for experts) be confused with the thermodynamic state of the cloud particles, i.e. whether they are liquid, mix-phased and solid.

- Further, sometimes you refer to the 'cloud state' as optical state of the cloud (compare Page 2, line 24 with page 3, line 26). Changing the notion in scientific paper is very dangerous since

a.) in order to avoid confusion the same thing should always have the same notion properly defined early on in a manuscript, and

b) the notion optical cloud state is not unambiguous since many small cloud particles (polluted clouds) as compared to few but large cloud particles (pristine clouds) would lead to a different cloud color (white vs grey respectively), also a form an optical cloud state.

- page 4, line 16: clouds to not 'switch' between think and thin states, but their optical thickness (and skylight radiance) has a deep routing in atmospheric dynamics, and thermodynamics.

- Page 5, line 5 - to 11: Define Lsc

- Page 6, line 18: thin/thick duality: I guess you mean the ambiguity in skylight radiance at $440 \mathrm{~nm}$ for thin and moderate thick cloud.

Printer-friendly version

- Page 6, line 30: What is a percent brightness? 
- Page 5, line 19: What is a 'spectral agility'? (choice of wavelength)

- Page 7, line 21: What is a 400nm long pass filter? (a band filter of a $400 \mathrm{~nm}$ wide AMTD band pass? starting at?).

- Page 9, line 21: What was averaged, and how?

- Page 10, lines 8 to $10: \ldots$ what is, and why prevented the secondary mission status of the (Aeronet) Cloud-Mode to infer COD of optically thin cloud covers?

- Page 10, line 29: What is photon noise? (photo electron shot noise?)

$-\ldots$.

- Finally, including a flow chart appears to be necessary in order to understand the various step involved in the data retrieval and reduction.

4. References The manuscript mostly lacks references to previous studies in the field. Some examples are given below:

- The introduction has no reference at all, which never occurred to me in the scientific literature.

- Page 4, Nose plot: Here you could easily refer to early work of King and Nakajima, or later studies of Marshak et al., and many others. Just inspect the list of reference in papers you cite or google for it....

- Page 5. When dwelling into the sensitivity of the inferred COD as function of ground albedo certainly the early work Kattawar, Melnikowa, Marskak, Chui and many others are worth to be cited...

- See above for Rodgers (2000).

Interactive comment on Atmos. Meas. Tech. Discuss., doi:10.5194/amt-2016-67, 2016. 